\title{
Patrones de asentamiento, rutas de comunicación y mercancías de intercambio a larga distancia en el Formativo Tardío del Austro Ecuatoriano
}

Modèles d'établissement, routes de communication et marchandises d'échange avec des territoires éloignés au Formatif Récent de la partie australe de l'Équateur

Settlement Patterns, Communication Routes and Items Involved in Long distance Trade in the Late Formative of Southern Highland Ecuador

\section{Karen Olsen Bruhns}

\section{(2) OpenEdition}

Journals

\section{Edición electrónica}

URL: http://journals.openedition.org/bifea/1871

DOI: $10.4000 /$ bifea. 1871

ISSN: 2076-5827

\section{Editor}

Institut Français d'Études Andines

Edición impresa

Fecha de publicación: 1 diciembre 2010

Paginación: 683-696

ISSN: 0303-7495

\section{Referencia electrónica}

Karen Olsen Bruhns, «Patrones de asentamiento, rutas de comunicación y mercancías de intercambio a larga distancia en el Formativo Tardío del Austro Ecuatoriano », Bulletin de l'Institut français d'études andines [En línea], 39 (3) | 2010, Publicado el 01 junio 2011, consultado el 08 diciembre 2020. URL : http://journals.openedition.org/bifea/1871 ; DOI : https://doi.org/10.4000/bifea. 1871

\section{(c) (†) $\odot$}

Les contenus du Bulletin de l'Institut français d'études andines sont mis à disposition selon les termes de la licence Creative Commons Attribution - Pas d'Utilisation Commerciale - Pas de Modification 4.0 International. 


\title{
Patrones de asentamiento, rutas de comunicación y mercancías de intercambio a larga distancia en el Formativo Tardío del Austro Ecuatoriano
}

\author{
Karen Olsen Bruhns*
}

\begin{abstract}
Resumen
En función de los datos actualmente disponibles, parece que el Periodo Formativo se atrasó en los Andes australes del Ecuador. Con el florecimiento de la cultura Chorrera, con sus necesidades de materiales exóticos, aparentemente una necesidad relacionada con un incremento en la estratificación social y el crecimiento de cacicazgos complejos, se pueden ver los comienzos de la cerámica, la agricultura (?), y, un poco más tarde, el pastoreo de camélidos. En las actuales provincias del Azuay y Cañar tenemos datos que indican el surgimiento de aldeas relativamente pequeñas, tanto en las vegas de los ríos como en la cima de las montañas. Estas aldeas manifiestan un alto grado de intercambio, tanto entre ellas mismas, como con la costa y la selva y con las culturas del norte del Perú. La investigación sobre recursos naturales importantes en este intercambio está poco desarrollada, pero hay indicaciones de que el cinabrio, el cristal de roca, el oro y otros metales y, probablemente, materiales perecedores como los textiles de lana de camélido han formado parte de este intercambio. El intercambio a larga distancia permitía también la circulación de ideas y tecnologías.
\end{abstract}

Palabras clave: intercambio intercultural, cinabrio, cristal de roca

* Profesora emérita del Departamento de Antropología de la Universidad Estatal de San Francisco. E-mail:kbruhns@sfsu.edu 
Modèles d'établissement, routes de communication et marchandises d'échange avec des territoires éloignés au Formatif Récent de la partie australe de l'Équateur

\section{Résumé}

Selon les données actuellement disponibles, il semble que la Période Formative se soit retardée dans les Andes australes d'Équateur. Avec le fleurissement de la culture Chorrera, et ses nécessités en matériaux exotiques, qui tire probablement son origine d'une stratification sociale croissante et de la croissance de chefferies complexes, l'on peut observer les débuts de la céramique, de l'agriculture, et un peu plus tard, de l'élevage de camélidés. Nous disposons d'informations en provenance des provinces modenes de l'Azuay et de Canar, quir indiquent lémergence de hameaux relativement petits, tant dans les vallés fétles des fleuves que sur le sommet des montagnes. Ces petits villages connaissent du Pérou Peu de recherches ont été effectuées sur les ressources naturelles importantes dans le cadre de ces échnos, mis il existe des indications selon lesquelles le cinabre le cristal de roche l'or d'autres métaux, et probabismes des matériaux périssable tels les tissus de laine de camélidé, faisaient partie de ces échanges Lescontacts entre teritoireśloignés permestientégalementa ciculation des ées et de la technologie.

Mots clés : échange interculturel, cinabre, cristal de roche

\section{Settlement Patterns, Communication Routes and Items Involved} in Long distance Trade in the Late Formative of Southern Highland Ecuador

\section{Abstract}

On the basis of current evidence it would appear that the Formative Period was delayed in the southern sierra of Ecuador. With the rise of the Chorrera culture, whose emergent elites had a need for exotic goods, we see the beginnings of ceramics, agriculture and, somewhat later, camelid pastoralism. From the modern provinces of Azuay and Canar we have evidence of small villages, located in various ecological zones, and often on major routes of communication. These villages maintained a high degree of contact among themselves, but also were entering into trade with the coast and the tropical forest to the east, presumably via routes which are largely still in use. The investigation of the materials involved in this trade has hardly begun, but sea shells, ceramics, cinnabar, rock crystal, colored hard stones, metals and probably perishable materials such as textiles of camelid wool were involved. Long

Key words: intercultural exchange, cinnabar, rock crystal

El Formativo Tardío del Ecuador es una época de grandes cambios culturales en la sierra austral (fig. 1). La cerámica aparece después de lo que parece haber sido un arcaico prolongado. Está bastante claro que las tradiciones cerámicas iniciales en el Austro están muy relacionadas con la tradición Chorrera de la costa. Mi hipótesis provisional es que las culturas chorreroides han estado en el proceso de una expansión de sus redes de comunicación con el fin de adquirir los materiales

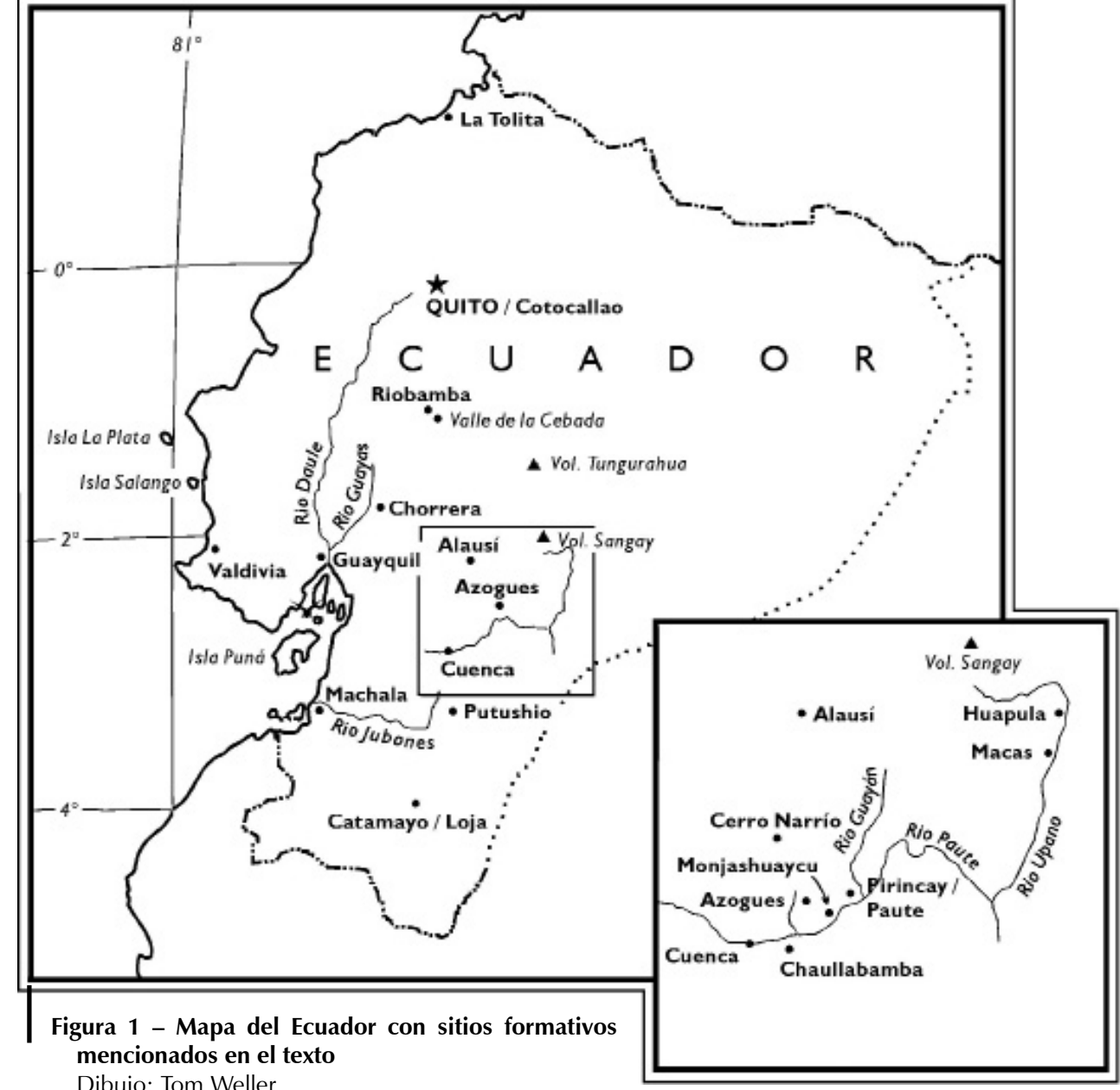

exóticos necesarios para sus élites. La ruta costera de intercambio con la costa norte del Perú y la que va hasta Colombia funciona desde los tiempos valdivianos pero, en el Formativo Tardío, más o menos cerca del 1400 a. C., las redes de intercambio entre la costa, la sierra austral y la selva se desarrollan de la forma que tendrán en el resto de la prehistoria.

Los pocos sitios australes de esta época ya excavados nos demuestran que, a pesar de la aparición de industrias especializadas para servir estos nuevos mercados, la mayoría de los sitios son pequeños. Es muy probable que la mayor parte de la población viva en casas aisladas o en aldeas muy pequeñas, de acuerdo a los patrones de asentamiento tradicionales en la región hasta hoy en día (fig. 2) (Bennett, 1946:14; Bruhns, 2003:148-153). 


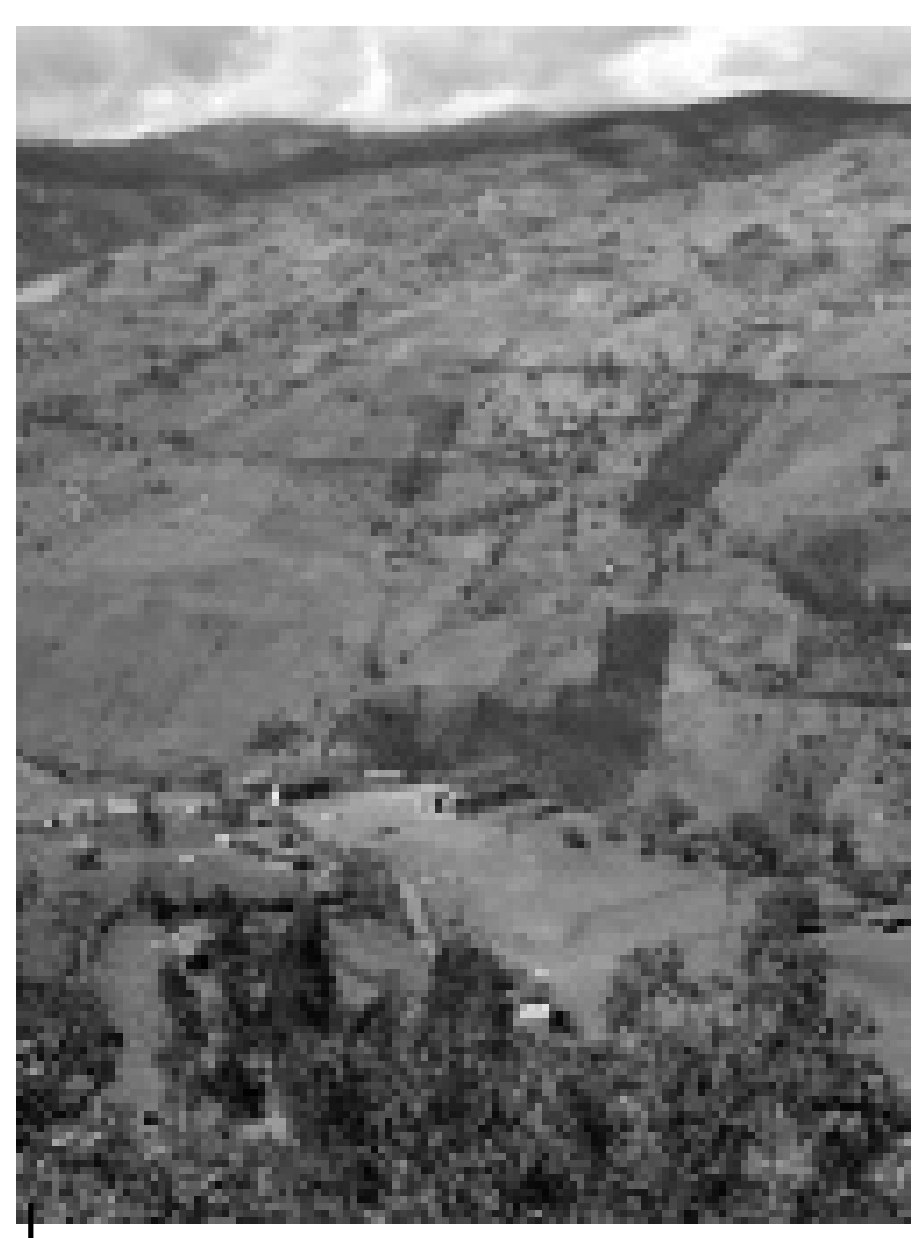

Figura 2 - Centro ceremonial moderno en la Cola de San Pablo (Azuay) Asentamientos aislados y aldeas pequeñas son típicas del Austro hast hoy en dí. Foto: K. Bruhns

\section{ASENTAMIENTOS $Y$}

\section{ARQUITECTURA}

No sabemos mucho de la arquitectura temprana en el Austro. Bennett especula que los edificios son pequeños y perecederos, probablemente construidos con madera y tejados de paja (1946: 14). En el sitio de Pirincay, el edificio más temprano es una plataforma baja de piedra y barro con un piso de barro duro. Sus asociaciones arqueológicas incluyen materiales de la costa. Las estructuras en los niveles superiores involucran el relleno y nivelación del sitio central, aparentemente con la finalidad de construir una serie de plazas pequeñas. Las primeras plazas se construyen de barro pero más tarde utilizan un pavimento de una cal (carbonato de calcio, $\mathrm{CaCo3}$ ), que aparece frecuentemente en la zona de forma natural (fig. 3). Esta cal posiblemente forma parte del intercambio a larga distancia. El hábito de chacchar coca aparece con la cultura Valdivia, aunque la historia de esta práctica en la sierra no esté tan clara (Ostenada \& Espíndol, 2004). Los «altares» de Uhle en Chaullabamba parecen haber sido pavimentos blancos similares a los de Pirincay (Uhle, 1922). Sin embargo, no existen muchas evidencias de superestructuras hasta más tarde en la secuencia estratigráfica, ni en Pirincay ni en Cerro Narrío, y no sabemos si han existido estructuras diferenciadas por el estatus de sus habitantes o por su función. Moldes de postes excavados por Gomis en Chaullabamba, un sitio cuya ocupación se restringe a los siglos más tempranos del Formativo Tardío Serrano, sugieren edificios rectangulares (Gomis, 1989; Idrovo, 1989). Restos de edificios muy similares se han encontrado en Pirincay y existen evidencias de muros de bajareque y techos de paja. Guffroy (1986) ha excavado una casa circular/ovoide en Loja. Esta casa tiene muros bajos de piedra y un techo grande de paja, indicando que evidentemente han existido algunas variaciones locales en la arquitectura serrana. La falta de excavaciones

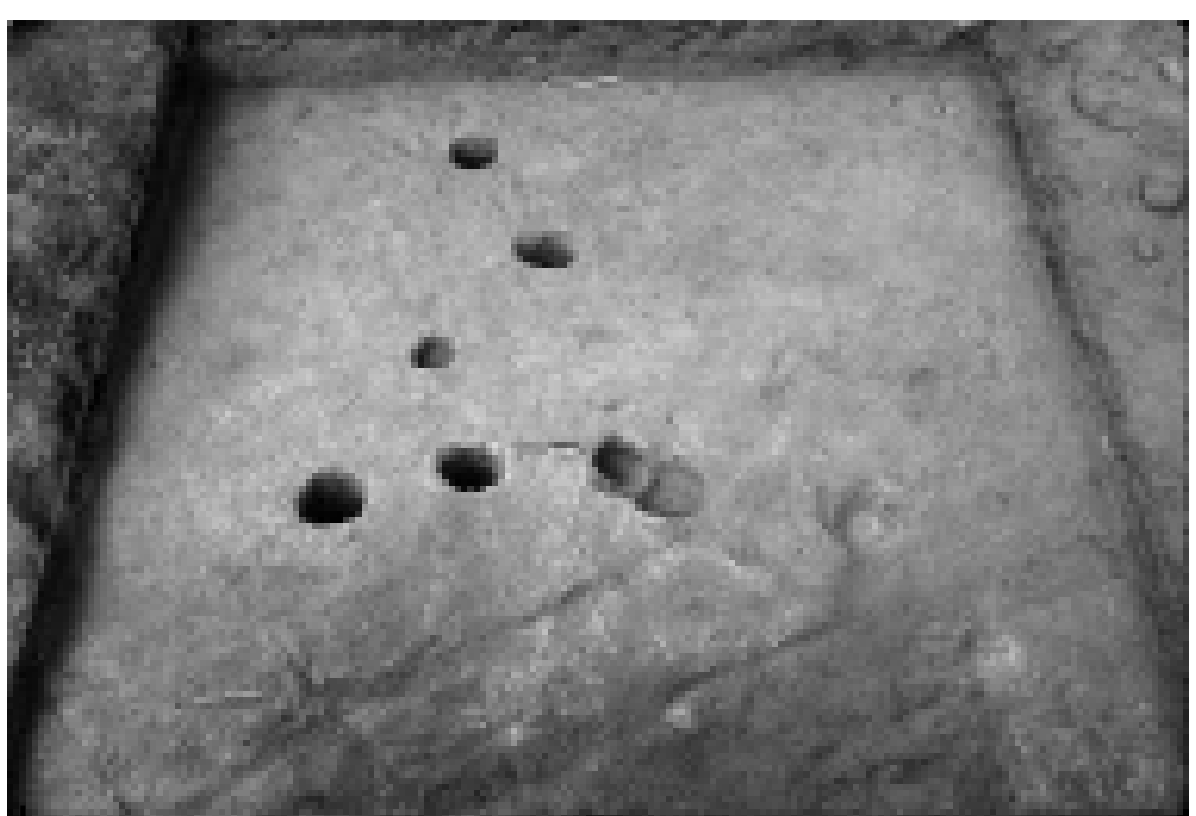

Figura 3 - Piso (iplaza?) blanco con moldes de poste de un edificio posterior en Pirincay Foto: K. Bruhns

dedicadas a la delineación de sitios y a la arquitectura en la sierra sur provoca muchas especulaciones respecto a la organización social/política de estas aldeas. Lo único evidente es que han sido muy activas en el intercambio de bienes entre ellos mismos y con áreas más alejadas al este y oeste.

\section{ORGANIZACIÓN DEL INTERCAMBIO Y RUTAS DE COMUNICACIÓN}

Falta mucha información acerca de la organización del intercambio a larga distancia y la comunicación entre entidades culturales en el Ecuador antiguo. Para el periodo prehispánico tardío tenemos evidencia de, por lo menos, tres modos diferentes para el movimiento de bienes: el sistema de distribución centralizada de los incas, utilizado en los terrenos que controlan; los mindaláes de la sierra norte y la liga de mercaderes de la costa que emplean las balsas marinas para el movimiento de sus mercancías (Salomon, 1977; Norton, 1973; Marcos, 2005). Se desconoce la antigüedad de cualquiera de estos modos de de movimiento de los materiales. Quizás el hecho de la presencia Wari en el Austro familiariza a los ecuatorianos con un sistema de control del Estado en el intercambio a larga distancia desde la mitad del primer milenio a. C., e incluso más temprano (Bruhns, 1998), pero se desconoce su modo de organización. 
En los sitios formativos de Austro la cerámica, la piedra tallada y todos los aspectos de la cultura material son muy similares, indicando no solamente el contacto de estos sitios con los chorrera de la costa, sino también el alto grado de intercambio entre ellos mismos (Bruhns, 2003). Tipos de cerámica como el Narrío Pulido y el Negro con Líneas Bruñidas, hallados en Pirincay, vienen de Cerro Narrío y otros sitios (todavía desconocidos) de la región, de acuerdo con unos análisis llevados a cabo por el Dr. James Burton en el Laboratorio de Química Arqueológica de la Universidad de Wisconsin. Sus análisis sugieren que lo importante es el contenido de estas vasijas o bien la interacción implícita en la utilización de las cerámicas. El intercambio de regalos es un medio común para el movimiento de materiales en sociedades igualitarias y la cantidad de objetos importados en estos sitios no es tan grande. Sin embargo, la existencia de talleres de cuentas de cristal de roca en Pirincay indica que se establece una producción especializada para el intercambio a larga distancia, en este caso el mercado costeño y que, tal vez, el proceso de diferenciación de sitios también esta en curso (fig. 4).

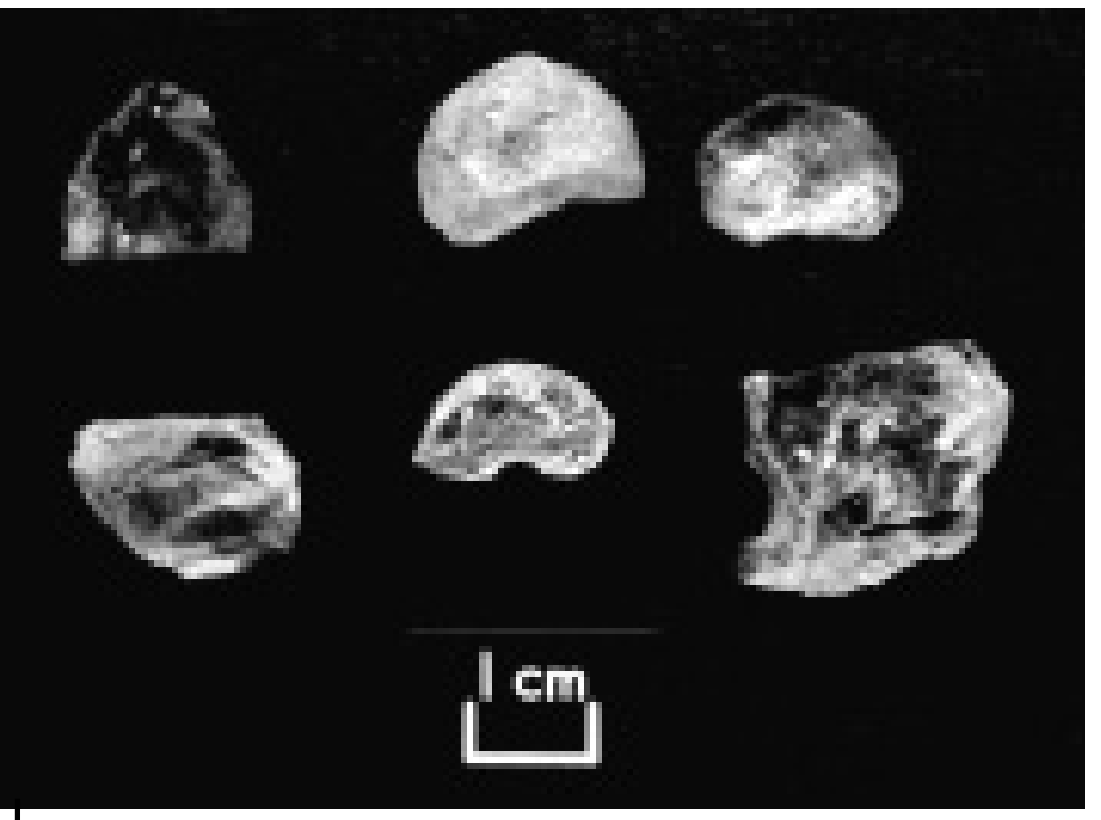

Figura 4 - Cuentas de cristal de roca en diversas etapas de producción (Pirincay) Foto: K. Bruhns

Los distintos sitios contienen cantidades muy diferentes de bienes importados: en Cerro Narrío, por ejemplo, hay cantidades grandes de concha Spondylus tallada (Collier \& Murra, 1943). En Chaullabamba parece haber más cerámica importada de la costa (Uhle, 1922; Gomis, 1989; Idrovo, 1989; Greider, 2009). Por su parte, Pirincay contiene bastante cerámica Chorrera de tipos diferentes, incluyendo una pieza que, posiblemente, pertenece a Piura (Bruhns, 2003).
Debido a la evidencia más tardía de interrelaciones muy próximas con las culturas peruanas norteñas, es muy probable que sea de ahí donde provenga la vasija de la que solamente queda un pequeño tiesto. No obstante, la cantidad total de materiales importados no es muy grande, indicando que un intercambio en cadena es una buena posibilidad, sobre todo si se intercala con un intercambio de regalos por medio de rituales, bodas u otras ocasiones. La introducción de los camélidos domesticados en el siglo $\mathrm{V} \mathrm{d}$. C. abre la vía para caravanas regulares, tal vez organizadas por familias y compañeros/parientes regulares en la ruta, como las caravanas modernas de Paratía en el Perú (Flores Ochoa, 1968).

Tenemos una única clase de evidencia acerca de quiénes estaban involucrados en el intercambio a larga distancia. ¿Ha sido gente de la costa viajando a la sierra o encontrad muy grande, pero distribuida a lo largo de la estratigrafía de casi mil doscientos años, de conchas marinas comunes. En su mayoría son ejemplares de anadara (ostra del manglar) pero también aparecen otras conchas pequeñas. No están modificadas ni son del tipo de conchas utilizadas para la talla de ornamentos o herramientas. Es muy probable que sean recuerdos, algo que un serrano, en el viaje de su vida hasta la costa, ha recogido para mostrar a sus hijos o solamente para tener como recuerdo de su aventura (fig. 5).

La rutas de intercambio presentan otro problema. Aunque existen bastantes vestigios de caminos precolombinos en el Austro, la mayoría no están adecuadamente cartografiados (esta situación puede cambiar con la llegada de los GPS) y en general se asumen todos como incaicos. Es posible que muchos daten de esa época, debido a la intensiva construcción de infraestructura en el Austro por los incas, pero gran cantidad de estos caminos están claramente asociados con sitios más tempranos. La ubicación de muchos de los sitios formativos ya conocidos - cercanos, en épocas más tardías, a rutas importantes a través o a lo largo de las montañas- nos sugiere que estas rutas son bastante antiguas. Cerro Narrío, por ejemplo, se ubica cerca de una ruta tradicional (y moderna) a través de la sierra a la cuenca de Riobamba y también a la ruta que llega desde la cuenca de Guayaquil por medio del río Cañar (Braun, 1973). Los sitios de los valles de los ríos Tomebamba y Paute se ubican en las rutas importantes hacia Cañar (por los valles de los ríos pero también por los páramos) y el valle del Paute ha sido una ruta de acceso muy importante desde el río Upano y el Alto Amazonas hasta la 
construcción del Centro Hidroeléctrico Paute en la Cola de San Pablo, cañón del Paute bajo en los años 1970. Loma Pucara se ubica en una ruta entre la sierra y la montaña oriental (Arellano, 1994). La ruta del río Jubones desde la sierra hasta la costa sur del Ecuador continúa siendo importante, así como la ruta a través del páramo hacia Molleturo y a la isla Puná (Carmichael et al., 1979).

\section{MATERIALES INVOLUCRADOS EN EL INTERCAMBIO CON ZONAS ALEJADAS}

También es necesario considerar qué tipos de bienes pasan entre zonas ecológicas y los grupos étnicos diferentes. Los productos serranos importantes en la costa son cuentas y ornamentos de piedras duras, coloradas y de cristal de roca. El cristal de roca viene de la formación «El Pan», la única fuente de este material en el Austro. Hasta ahora únicamente hemos identificado un local de talla de cuentas, en el sitio de Pirincay, a unos 15 kilómetros río arriba desde la desembocadura del río Collay en el Paute. La quebrada del río Collay está llena de guijarros de cristal de roca, producto de la erosión de las vetas de cuarzo de los lados (Burton, n.d.; Bruhns, 1991). En Pirincay hemos identificado más de 45 talleres, desde las capas más profundas hasta la superficie del sitio, donde se han identificado los restos de la producción de cuentas semejantes a los hallados en los sitios chorreroides. El tamaño de los pequeños talleres sugiere una producción artesanal de las cuentas por individuos, mas no a gran escala. La calcedonia roja y verde, la toba rojiza y la pizarra blanca, todas utilizadas para cuentas, colgantes, ucuyayas, u otros ornamentos, tanto en la sierra como en la costa, tienen una distribución amplia en el Austro, así como las otras piedras verdes, incluyendo varios tipos de metagreywackes y serpentinas. Existen depósitos de turquesa en el valle del río Jubones, aunque su ubicación exacta es desconocida. La cal es abundante en el valle del río Paute. El cinabrio, una sustancia importante en casi todas las culturas ecuatorianas y peruanas, se halla en Cañar. Las fuentes de cinabrio de Loma Guashon (Azogues) y Cañaribamba se conocían en la antigüedad, a juzgar por los hallazgos de este material en Pirincay. Las minas de cinabrio, sobre todo las de Azogues, son muy productivas en el periodo colonial temprano. Las minas de Loma Guashon producen tanto que su cinabrio Figura 6 - Loma Guashon, cerca Azogues, Cañar
Las minas de cinabrio más productivas de la llega a México (Chacón, 1986: 18-19; Truhan et al., 2005) (fig. 6). El oro del río Santa Bárbara, importante desde los tiempos de los wari hasta el presente $y$, probablemente también en una época más temprana, puede haber sido un producto indispensable hacia el final del Formativo Tardío. También en Oña y el norte de Loja, Matilde Temme ha identificado una minería formativa en el sitio de Putushío. Del mismo modo, existen fuentes de oro abundantes en la costa, aunque no sabemos cuándo empezó su explotación.

¿Qué quieren o reciben los pueblos serranos? La concha Spondylus. Esta concha anaranjada aparece en todos los sitios formativos de la sierra austral en el Formativo Tardío. La concha parece haber llegado ya elaborada;

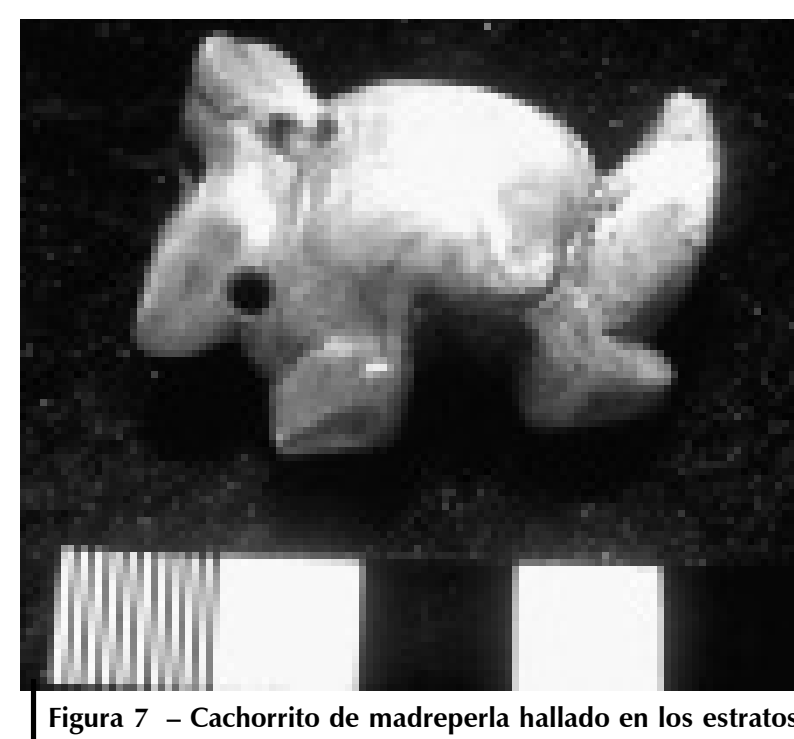

más profundos de Pirincay Foto: K. Bruhns

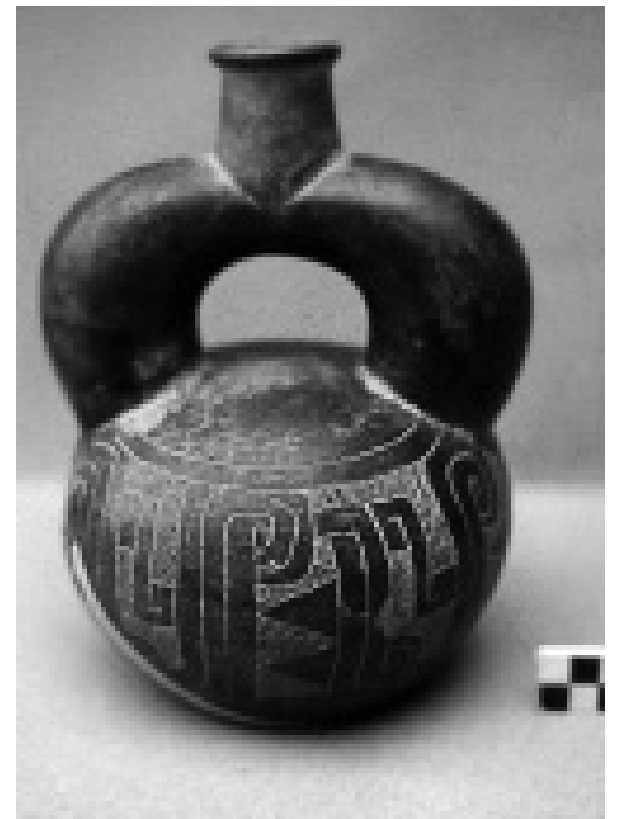

Figura 8 - Botella hallada en El Descanso a la junción de los ríos Tomebamba Azogues

Museo del Banco Central del Ecuado Cuenca. Foto: K. Bruhns no existen evidencias de la talla en estos sitios. La madreperla (Pinctada spp.) también tiene un valor alto para los serranos así como otras conchas Strombus o el Lyropecten. Todas estas conchas aparecen en las capas más tempranas de Pirincay (fig. 7).

La cerámica Chorrera también es importante en este intercambio. Se ha identificado esta cerámica en Cerro Narrío, Chaullabamba, Pirincay y probablemente en Monjashuaycu y Huancarcucho. La mayoría de las piezas Chorreras son vasijas modeladas, botellas de asa estribo, como la famosa botella incisa de El Descanso, o figurillas (fig. 8). También son comunes cuencos sencillos con pintura iridiscente, tanto gris como rojiza, iguales a piezas de la península de Santa Elena.

Hay otros materiales importantes en el intercambio entre zonas alejadas que no dejan sus huellas en los suelos arqueológicos de la sierra. La sal del mar es un producto de importancia debido a la falta de salinas en la sierra (Pomeroy, 1985).

En tiempos históricos la sal del Austro proviene de la Isla Puná, un lugar importante en la industria de la obtención de sal de mar. Los tejidos de algodón también son una mercancía que puede haber sido 
muy deseada. El algodón puede crecer solamente en dos áreas muy restringidas del valle del Paute — es también necesario obtenerlo de la costa o la selva-. Los tintes también han debido mantener similar suerte. La producción de añil empezó en el Perú hacia 2500 a. C. En el Ecuador no tenemos evidencias concretas del añi hasta el Periodo del Desarrollo Regional cuando aparece en la pintura poscocción de figurillas de las tradiciones Jama-Coaque y Bahía. Pero, teniendo en cuenta las evidencias de intercambio con la costa norte del Perú desde tiempos valdivianos, y también la importancia de los tejidos en todas las sociedades andinas, es muy probable que el cultivo de añil empezara temprano, tanto en el Ecuador como en el Perú. Las plantas de las que se alimenta la cochinilla y otras que dan tintes rojos como Relbunium y Arribidea chica pueden cultivarse tanto en la sierra como en la costa.

El pescado seco puede haber sido otra mercancía. El pescado, seco o salado, ha sido un capítulo fundamental en el intercambio entre la costa y la sierra hasta la invención de congeladores y máquinas para el hielo que permiten la importación de pescado fresco (por medio de camiones).

Para la selva, tenemos muy pocas evidencias de intercambio, aparte de las hachas en forma de $\mathrm{T}$, hasta los comienzos de Desarrollo Regional cuando aparece la cerámica Incisa de Franjas Rojas, una cerámica que se fabrica en los alrededores de Macas. Esta cerámica se halla en la mayoría de los sitios formativos de la selva (Bruhns et al., 1994; Porras, 1971; Arellano, 1994). Hay que destacar una cerámica más temprana, hallada en Chaullabamba e identificada como Incisa de Franjas Rojas por Greider. No guarda vinculación con la cerámica de Macas, identificada por Bushnell en 1946 y estudiada por Rostocker (1996). Esta identificación es muy problemática porque la cerámica descrita por Greider (2009: 69-72) no proviene de la selva sino que es un estilo local, un subestilo menor del conjunto del Formativo Tardío que se halla a lo largo del Austro.

En su gran mayoría los productos selváticos son perecederos. Materiales como las plumas y pieles de animales pueden haber sido importantes para los serranos, aunque probablemente los costeños hayan logrado conseguir esos objetos de áreas más cercanas como la ancha costa tropical del Ecuador. La miel de abejas y la cera también deben haber sido importantes, sobre todo cuando la metalurgia apareció en Oña hacia el final del Formativo y en el Azuay no mucho más tarde (Rehren \& Temme, 1994; Bruhns, 1994). También se cultiva algodón en la selva, algo de interés para los serranos. Los curanderos y las drogas son otros elementos importantes de la selva. Recordemos a los kallawaya de Bolivia, curanderos viajeros muy importantes en los Andes que probablemente tengan sus raíces en las épocas prehispánicas (Bastien, 1987). Michael Harner documenta también la importancia de los chamanes selváticos en el Ecuador y habla de sus viajes (Harner, 1972).

¿Qué quería la gente de la selva? Hallazgos recientes en Mayo-Chinchipe nos demuestran que esta gente del Formativo quiere la concha Spondylus y piedras duras coloradas, como la calcedonia roja, verde y turquesa. Aunque Valdez (2007) plantea la hipótesis de una importante fuente todavía desconocida de turquesa selvática, sabemos que existen las fuentes del río Jubones y probablemente existan otras fuentes serranas, sobre todo en Loja, por su riqueza de metales. Los hallazgos del padre Porras en la Cueva de los Tayos aportan valvas grandes de Spondylus y unas 50 cuentas y colgantes de Madreperla y Conus (Porras, 1978; 1987: 230). Fuera de estos hallazgos solamente podemos especular. A la vez, debemos recordar que el intercambio puede darse entre gentes de áreas con los mismos recursos, con la finalidad de fomentar la cohesión social, tal como lo muestra Chagnon para los yanomami (1968). Sin embargo, bienes especializados, como tejidos decorados, cerámicas, ornamentos y, por supuesto, las ideologías, danzas, canciones, rituales, y otros productos no tangibles, continúan formando parte importante del intercambio entre grupos tradicionales hasta el día de hoy.

El intercambio con el Perú, con los grupos costeros como intermediarios o directamente, se nota en la llegada de maíz de tipo peruano a los niveles profundos de Pirincay. El maíz de los años finales del Formativo es de un tipo similar al de Cotocallao, probablemente reflejo más de un contacto intenso con la sierra norte. En estos niveles también hallamos la poca obsidiana encontrada en el sitio, que viene de las fuentes de Mullumica (Burger et al., 1994). Tanto la metalurgia como los camélidos aparecen hacia finales del Formativo en Pirincay y, probablemente, en otros sitios de la sierra sur, seguramente importados y adoptados del Perú, pero no sabemos lo que llega desde el Austro hacia las culturas peruanas ni sus mecanismos de difusión.

\section{CONCLUSIONES}

A pesar de la escasez de investigaciones arqueológicas científicas en el Austro es evidente que el intercambio a larga distancia es importante desde los comienzos de la vida sedentaria y la introducción de la agricultura y la cerámica. El interés de las culturas chorreroides por los materiales exóticos de la sierra sur, y también muy probablemente por algunos productos selváticos, fomenta varios tipos de intercambio activo. Es probable que gran parte del intercambio en la sierra misma fuera intercambio en cadena y de regalos. Sin embargo, algunos hallazgos en Pirincay indican que gente serrana ha visitado la costa y, aunque las cantidades de materiales costeños no perecederos no son muy abundantes en los sitios serranos, sí son significativas. El pequeño tamaño de los sitios formativos serranos también indica que estamos hablando de cacicazgos muy sencillos y muy probablemente sociedades igualitarias en la mayor parte de los casos. Con la llegada de los camélidos del Perú, otra evidencia de dicho intercambio, es posible que apareciera un contacto más especializado. Son necesarias más investigaciones y análisis, tanto en la costa como en el Austro, para poder especificar cuál fue el rol del intercambio entre estas sociedades tempranas del Ecuador. 


\section{Referencias citadas}

ARRELLANO, J. G., 1994 - Loma Pucara. A Formative Site in the Cebadas Valley, Ecuador National Geographic Research \& Exploration, 10 (1):118-120.

BASTIEN, J., 1987 - Healers of the Andes: Kallawaya Herbalists and their Medicinal Plants, 198 pp.; Utah: University of Utah Press.

BENNETT, W. C., 1946 - Excavations in the Cuenca Region, Ecuador, 84 pp.; New Haven: Yale University Publications in Anthropology n. 35.

BONAVIA, D., 2008 - The South American Camelids, 628 pp.; LA: University of California. Cotsen Institute of Archaeology Press. Monograph n. ${ }^{\circ} 64$.

BRAUN, R., 1982 - The Formative as Seen from the Southern Ecuadorian Highlands. In: Primer Simposio de Correlaciones Antropológicas Andino-Mesoamericano (J. G. Marcos \& P. Norton, eds,): 41-100; Guayaquil: Escuela Superior Politécnica del Litoral.

BRUHNS, K. O., 1987 - Los talleres de cristal de roca de Pirincay, provincia del Azuay y el intercambio entre la sierra y la costa en el Formativo Tardío. Miscelánea Antropológica Ecuatoriana, 7: 91-100; Quito/Guayaquil.

BRUHNS, K. O., 1989 - Intercambio entre la costa y la sierra en el Formativo Tardío: nuevas evidencias del Azuay. In: Relaciones Interculturales en el Área Ecuatorial del Pácifico Durante la Epoca Precolombina (J.-F. Bouchard \& M. Guinea, eds.): 57-74; Oxford: B.A.R. International Series 503.

BRUHNS, K. O., 1991 - Los tallares de cristal de roca en Pirincay, provincia del Azuay. Miscelánea Antropológica Ecuatoriana, 7: 91-100; Guayaquil/Quito

BRUHNS, K. O., 1994 - Las culturas peruanas y el desarrollo cultural en los Andes septentrionales. Revista Memoria, 4: 251-267; Quito: MARKA.

BRUHNS, K. O., 1998 - Huaquería, procedencia y fantasía: el caso de los soles de oro del Ecuador. Boletín del Museo del Oro, 44-45: 183-205; Bogotá

BRUHNS, K. O., 2003 - Social and Cultural Development in the Ecuadorian Highlands and Eastern Lowlands During the Formative. In: The Ecuadorian Formative (S. Raymond \& R. L. Burger, eds.): 125-176; Washington D.C.: Dumbarton Oaks.

BRUHNS, K. O., 2004 - Llamas y rituales en el Formativo Tardío de la sierra austral del Ecuador. In: Simbolismo y ritual en los Andes Septentrionales (M. Guinea, ed.): 69-90; Quito/Madrid: Abya-Yala, Editorial Universidad Complutense.

BRUHNS, K. O., 2007 - Cerro Narrío, Pirincay y el Formativo Ecuatoriano. In: Reconocimiento y Excavaciones en el Sur del Ecuador (D. Collier \& J. Murra, eds.), apéndice D: 351-402; Cuenca: Casa de la Cultura Ecuatoriana, Núcleo del Azuay.

BRUHNS, K. O., BURTON, J. \& ROSTOCKER, A., 1994 - La Cerámica «Incisa en Franjas Rojas» evidencia del intercambio entre la sierra y el oriente durante el formativo tardío del Ecuador. In: Tecnología y Organización de la Producción de Cerámica Prehispánica en los Andes (I. Shimada, ed.): 53-66; Lima: Fondo Editorial de la Pontificia Universidad Católica del Perú.

BURGER, R. I., ASARO, F., SALAZAR, E., MICHEL, H. V. \& STROSS, F. H., 1994 - Ecuadorian Obsidian Sources Used for Artifact Production and Methods for Provenience Assignment. Latin American Antiquity, 5 (3): 257-277.

BURTON, J. H., s.f. - Pirincay Postdata; Madison: Universidad de Wisconsin. Manuscrito archivado en el laboratorio de Química Arqueológica.

BUSHNELL, G. H. S., 1946 - An Archaeological Collection from Macas, on the Eastern Slopes of the Ecuadorian Andes. Man, 46 (2): 2-6; London.
CARMICHAEL, E., WARWICK, B. \& ERICKSON, J., 1979 - Informe Preliminar de las nvestigaciones Arqueológicas en el Área de Minas, Río Jubones, Ecuador. Revista de Antropología, Sección de Antropología y Arqueología del Núcleo del Azuay de la Casa de la Cultura Ecuatoriana, 6: 130-153; Cuenca.

CHACÓN, J., 1986 - Historia de la minería en Cuenca; Universidad de Cuenca.

CHAGNON, N., 1968 - Yanomamö: The Fierce People; New York: Holt Rinehart Winston.

COLLIER, D. \& MURRA, J. V., 1943 - Survey and Excavations in Southern Ecuador; Chicago. Anthropological Series, Field Museum of Natural History, vol. 35.

CORDERO, L., 1950 - Enumeración Botánica. Provincias del Azuay y Cañar; Madrid: Afrodisio Aguado.

FLORES OCHOA, J., 1968 - Los pastores de Paratía; una introducción a su estudio, 159 pp. México: Instituto Indigenista Interamericana.

GOMIS, D., 1989 - La alfarería de Chaullabamba. Catedral Salvaje, 24: 4-5; Cuenca.

GREIDER, T., 2009 - Art and Archaeology of Chaullabamba, Ecuador; Austin: University of Texas Press.

GUFFROY, J. (ed.), 1986 - Loja Préhispanique. Recherches Archéologiques dans les Andes Méridionales de l'Équateur, 342 pp.; París: Éditions Récherche sur les Civilisations, «Synthese» n. ${ }^{\circ} 27$

HARNER, M. J., 1972 - Jivaro: people of the sacred waterfalls, 233 pp.; New YorK: Doubleday, Anchor.

IDROVO, U. J., 1989 - Chaullabamba: una ventana hacia nuestro pasado. Catedral Salvaje, 24: 2; Cuenca.

MARCOS, J. G., 2005 - Los pueblos navegantes del Ecuador Prehispánico, 206 pp.; AbyaYala; Quito.

NORTON, P., 1973 - El señorío de Sanangone y la liga de mercaderes: el cartel Spondylusbalsa. Miscelánea Antropológica Ecuatoriana, 6: 131-144.

POMEROY, C., 1985 - The Salt of Highland Ecuador: Precious Product of a Female Domain. Ethnohistory, 35 (2): 131-160.

PORRAS, P., 1977 - Fase Alausí. In: Estudios Arqueológicos. Antilles y Tierras Bajas de Sudamérica, Ecuador (P. I. Porras, ed.): 89-160; Quito: Ediciones de la Universidad Católica.

PORRAS, P., 1978 - Arqueología de la Cueva de los Tayos, 83 pp.; Quito: Ediciones de la Universidad Católica

PORRAS, P., 1987 - Nuestro Ayer. Manual de Arqueología Ecuatoriana; Quito: Centro de Investigaciones, Pontificia Universidad Católica del Ecuador.

OSTENADA, S. \& ESPÍNDOL, G., 2004 - El Uso de la Coca en el Antiguo Ecuador; Quito: Museo del Banco Central.

REHREN, T. \& TEMME, M., 1994 - Pre-Columbian Gold Processing at Putushio, South Ecuador: The Archaeological Evidence. In: Archaeometry of Pre-Columbian Sites and Artifacts (D. A. Scott \& P. Meyers, eds.): 267-284; California: The Getty Conservation Institute.

ROSTOCKER, A. G., 1996 - An Archaeological Collection from Eastern Ecuador; San Francisco: Department of Anthropology, San Francisco State University. Treganza Museum Papers Volume 18

SALOMON, F., 1977 - Pochteca and Mindalá: A comparison of Long-Distance Traders in Mesoamerica and Ecuador. Journal of the Steward Anthropological Society, 9 (1/2): $231-246$ 
TRUHAN, D., BURTON, J. \& BRUHNS, K. O., 2005 - El Cinabrio en el Mundo Andino. Revista de Antropología, 18: 193-206; Cuencua: Sección de Antropología y Arqueología del Núcleo del Azuay de la Casa de la Cultura Ecuatoriana.

UHLE, M., 1922 - Influencias mayas en el alto Ecuador; Quito: Tipografía y Encuadernación Salesiana.

VALDEZ, F., 2007 - Mayo-Chinchipe: The Half-Open Door. In: Ecuador: The Secret Art of Precolumbian Ecuador (D. Kelin \& I. Cruz Cevallos, eds.): 320-349; Milano: 5 Continentes. 